\title{
$18 \beta$-glycyrrhetinic acid exhibits potent antitumor effects against colorectal cancer via inhibition of cell proliferation and migration
}

\author{
SAISAI WANG ${ }^{1 *}$, YONG SHEN $^{2 *}$, RUNFENG QIU $^{1}$, ZHILIANG CHEN $^{1}$, ZHEHANG CHEN $^{3}$ and WENBIN CHEN ${ }^{1}$ \\ ${ }^{1}$ Department of Colorectal Surgery, The First Affiliated Hospital, Zhejiang University School of Medicine, Hangzhou, \\ Zhejiang 310003; ${ }^{2}$ Key Laboratory of Cancer Prevention and Intervention, China National Ministry of Education, \\ The Second Affiliated Hospital, Cancer Institute, Zhejiang University School of Medicine, Hangzhou, \\ Zhejiang 310058; ${ }^{3}$ Zhejiang University School of Medicine, Hangzhou, Zhejiang 310058, P.R. China
}

Received November 14, 2016; Accepted June 13, 2017

DOI: 10.3892/ijo.2017.4059

\begin{abstract}
Accumulating evidence shows that $18 \beta$-glycyrrhetinic acid (GRA) has antitumor activities in breast, ovarian cancer and leukemia, while its role in colorectal cancer remains unknown. In the present study, we investigated the effect of GRA in colorectal cancer cells LoVo, SW480 and SW620 and studied the underlying molecular mechanisms. Results showed that GRA had potent inhibitory effects on colorectal cancer cell proliferation in a dose- and time-dependent manner in vitro and in vivo. Growth inhibition was mediated by pro-apoptosis, as evident from Annexin V-FITC staining, the reduced expression of survivin and the induced expression of cleaved PARP. Furthermore, GRA treatment resulted in marked reduction of cell migration, invasion and wound healing capability, accompanying by the downregulated MMP expression. Moreover, GRA decreased the protein levels of p-PI3K, p-AKT, p-STAT3, p-JNK, p-p38 and p-NF-кB p65, of which the phosphorylation of PI3K and STAT3 decreased as early as $2 \mathrm{~h}$ after the GRA treatment. These results suggest that regulation of the apoptosis, invasion and migration of colorectal cancer cells by GRA might be through suppressing PI3K and STAT3 signaling pathways. The present study indicated that GRA could be a potential effective therapy for patients with colorectal cancer.
\end{abstract}

Correspondence to: Dr Wenbin Chen, Department of Colorectal Surgery, The First Affiliated Hospital, Zhejiang University School of Medicine, 79 Qingchun Road, Hangzhou, Zhejiang 310003, P.R. China

E-mail: cwbin@hotmail.com

*Contributed equally

Key words: $18 \beta$-glycyrrhetinic acid, colorectal cancer, apoptosis, migration, invasion, MMP, p-PI3K, p-STAT3

\section{Introduction}

Radix Glycyrrhiza, the most extensively-used ingredient in formulas of Chinese medicine, has been considered to treat diseases (including cancers) for thousands of years in China. The $18 \beta$-glycyrrhetinic acid (GRA) (Fig. 1), a pentacyclic triterpenoid extracted from the root of Licorice, has been shown to display multiple physiological functions, such as anti-microbial $(1,2)$, anti-inflammatory $(3,4)$, anti-viral $(5)$, anti-ulcer (6) and hepatoprotective effects (3). Recently, the antitumor effect of GRA has been extensively investigated in vitro and in vivo in breast $(7,8)$, ovarian cancer (9), promyelocytic leukemia (10), hepatoma and stomach cancer cells (11). The mechanisms of antitumor of GRA include disruption of actin cytoskeleton (12), inhibition of metastasis by impairing the p38 MAPK-AP1 signaling axis (7) and induction of Fas- or DNA fragmentation-mediated apoptosis (9-11). Furthermore, GRA has selective toxicity against tumor cells while has no effect on normal cells and its efficacy is more potent than those of some clinically available antitumor agents (12).

Colorectal cancer (CRC) is the third most commonly diagnosed cancer and the fourth cause of cancer-related deaths worldwide (13). Colorectal cancer presents multiple processes involving accumulation of mutations in oncogenes, inactivation of tumor suppressors and existence of various signaling pathways, leading to genetic disorders, dysregulation of apoptosis, increased invasiveness and morphological progression (14). Current therapy of colorectal cancer involves surgery, chemotherapy, radiotherapy and targeted therapy. Chemotherapy helps impeding cell growth, triggering cell death, preventing cancer cell metastasis and lowering the risk of recurrence $(15,16)$. While chemotherapy is money-consuming and has many side-effects such as myelosuppression, fatigue, pain, diarrhea and so on. Alternative effective therapies are in demand. Traditional Chinese medicine (TCM) is an available option which has the advantage of reducing toxic side-effects and improving the efficacy of chemotherapy $(17,18)$.

Although the antitumor effects of GRA have been extensively studied, its role in colorectal cancer and the underlying mechanisms remains unknown. In the present study, we report 


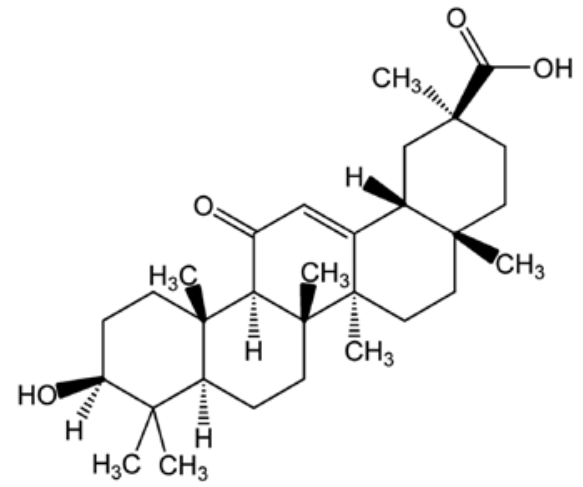

Figure 1. Chemical structure of $18 \beta$-glycyrrhetinic acid (GRA).

that GRA can inhibit the proliferation of colorectal cancer cells both in vitro and in vivo which might be through the induction of pro-apoptosis. We also found that GRA potently inhibits the migration and invasion of colorectal cancer cells and reduces matrix metalloproteinase (MMP) expression as well. Furthermore, our findings suggested the regulation of apoptosis, invasion and migration of colorectal cancer cells by GRA probably through suppressing PI3K and STAT3 signaling pathways.

\section{Materials and methods}

Reagents and antibodies. $18 \beta$-glycyrrhetinic acid (98\% of purity, determined by HPLC) was purchased from Aladdin Bio-Chem Technology Corp. (Shanghai, China) and dissolved in dimethyl sulfoxide (DMSO). Antibodies used in western blotting against MMP1, MMP2, MMP3 and MMP9 were bought from Proteintech Group Inc. (Chicago, IL, USA). Antibodies against Poly(ADP-ribose) polymerase (PARP), phospho-PI3K p85/p55, phospho-AKT, AKT, phospho-STAT3, STAT3, phospho-p38, p38, phospho-Erk1/2, Erk1/2, phosphoSAPK/JNK, SAPK/JNK, phospho-NF- $\mathrm{B}$ p65, NF- $\mathrm{B}$ p65 and $\beta$-actin were obtained from Cell Signaling Technology (Danvers, MA, USA).

Cell culture. The human CRC cell lines LoVo, SW480 and SW620 and a normal human colon mucosal epithelial cell line (NCM460) were obtained from the American Type Culture Collection and cultured in RPMI-1640 medium complemented with $10 \%$ heat-inactivated fetal bovine serum (FBS), penicillin/streptomycin $(100 \mathrm{U} / \mathrm{ml})$ and L-glutamine $(2 \mathrm{nM})$. Cells were cultured at $37^{\circ} \mathrm{C}$ with $5 \% \mathrm{CO}_{2}$ in a humid incubator. All the cell culture reagents were purchased from Invitrogen (Shanghai, China).

Cell growth inhibition assay. Cells were plated in 96-well plate at a density of $1 \times 10^{3}$ cells/well and cultured in complete medium supplemented with varied concentrations of GRA $(0,12.5,25$ and $50 \mu \mathrm{M})$. After culturing for $24,48,72$ and $96 \mathrm{~h}$, cell growth was analyzed by CCK-8 assay. A total of $10 \mu \mathrm{l}$ of CCK-8 solution reagent (Beyotime Institute of Biotechnology, Haimen, China) was added to each well and cultured for another $1 \mathrm{~h}$. Absorbance at $450 \mathrm{~nm}$ was measured on an ultra-microplate reader (EMax; Molecular Devices,
Sunnyvale, CA, USA). The growth inhibition rate was calculated with the following formula: (Ab. control - Ab. treated)/ $\mathrm{Ab}$. control $\mathrm{x} 100$. The $\mathrm{IC}_{50}$ was taken as the concentration at which it caused $50 \%$ inhibition of cell proliferation $(50 \%$ reduction in the absorbance value in the treated cells, in respect to control). $\mathrm{IC}_{50}$ was calculated by curve fitting statistical analysis by GraphPad Prism (Graphpad Software, Inc., San Diego, CA, USA).

Annexin V apoptosis assay. To determine apoptosis, the cells were stained with the Annexin V apoptosis kit according to the manufacturer's instructions (Lianke Biotech, Co., Ltd., Hangzhou, China). In brief, $1 \times 10^{5}$ cells in 6 -well plate were treated with or without GRA for $48 \mathrm{~h}$ before cells were trypsinized, washed with ice-cold PBS, resuspended in $500 \mu 1$ binding buffer and incubated with $5 \mu \mathrm{l}$ Annexin V-FITC and $10 \mu \mathrm{l}$ propidium iodide (PI) working solution at room temperature for $5 \mathrm{~min}$ in the dark. Stained cells were then analyzed by flow cytometry (BD FACScan; BD Biosciences, San Jose, CA, USA).

Reverse transcriptase-quantitative polymerase chain reaction $(R T-q P C R)$. Cells in 6-well plate at a density of $2 \times 10^{5}$ were treated with GRA at different concentrations for $24 \mathrm{~h}$ and total RNA was extracted using Ultra-Pure RNA kit (Cwbiotech, Beijing, China). After DNase treatment, cDNA was synthesized by the HiFiScript cDNA Synthesis kit (Cwbiotech). Reverse transcriptase PCR was performed on 2720 Thermal Cycler (Applied Biosystems, Foster City, CA, USA). Quantitative PCR was then performed with $1 \mu \mathrm{l}$ of cDNA using the iTaq Universal SYBR-Green Supermix (Bio-Rad Laboratories) on CFX96 Touch Real-Time PCR Detection System (Bio-Rad Laboratories). The specific primers for survivin and $\beta$-actin were as follows: survivin: 5'-GATTTGA ATCGCGGGACCCGTTG-3' (forward) and 5'-TCAAGAC AAAACAGGAGCACAGT-3' (reverse); $\beta$-actin: 5'-GTATCC TGACCCTGAAGTACC-3' (forward) and 5'-TGAAGGTCTC AAACATGATCT-3' (reverse). qPCR results were analyzed with Bio-Rad CFX Manager (Bio-Rad Laboratories). A relative quantification delta CT strategy was used to analyze the qPCR data. The calculated threshold cycle was normalized to the value of internal $\beta$-actin amplified from the same cDNA and the fold-change was calculated as referenced to control.

Migration and invasion assay. Transwell migration and invasion assays were carried out using cell culture chambers with 8- $\mu \mathrm{m}$ pore filter (Corning Costar Corp., Cambridge, MA, USA). For invasion assay, the upper surfaces of the membranes were coated with $50 \mu \mathrm{l}$ Matrigel (BD Biosciences) $6 \mathrm{~h}$ before cells were seeded. Cells/well $\left(2 \times 10^{5}\right)$ in $200 \mu \mathrm{l}$ serum-free RPMI-1640 were added into the upper chamber of 24-well plates supplied with or without $50 \mu \mathrm{M}$ GRA. Culture media containing $20 \%$ FBS were added in the lower chamber. After $48 \mathrm{~h}$ culturing, the cells in the upper chamber were removed by wiping the upper side of the membrane with a cotton swab and cells in the lower surface of the membrane were fixed with icecold methanol for $5 \mathrm{~min}$ and stained with crystal violet in $20 \%$ ethanol overnight. Images were acquired by light microscopy and the cells invaded to the underside of the membrane were 
quantitated by cell counting in five predetermined fields. To determine whether the invasion inhibition was caused by the decreasing of cells number, the number of the LoVo cells after invasion was recorded in both sides of the chamber.

The methods used for the migration assay were almost the same as the invasion assay described above, except for no Matrigel coating in the upper surfaces of the membranes.

Wound healing assay. Cells were seeded in 24-well plate at an initial density of $1.5 \times 10^{5} /$ well and allowed to grow to $90 \%$ confluency in complete medium. Cells were wounded by a plastic tip and then washed three times with phosphate-buffered saline (PBS) to remove the cell debris and incubated for $24 \mathrm{~h}$ in $5 \%$ FBS medium containing different concentrations of GRA. Images of the wound morphology were acquired by light microscopy.

Xenograft tumor models. Four- to five-week-old female $\mathrm{BALB} / \mathrm{c}$ nude mice, weighing between 16-18 $\mathrm{g}$ were purchased from Shanghai Laboratory Animal Company (SLAC; Shanghai, China). Mice were maintained in the animal facility at the Zhejiang University, China. The animal experiments were approved by Animal Care and Use Committee of Zhejiang University. LoVo cells $\left(5 \times 10^{6}\right)$ resuspended in $100 \mu 1$ PBS were injected subcutaneously in the right flanks of the nude mice. When tumors reached an average volume of $100 \mathrm{~mm}^{3}$, the mice were randomly divided into two groups. Mice in GRA-treated group were intraperitoneally injected with GRA at $40 \mathrm{mg} / \mathrm{kg}$ once every 2 days in $1 \%$ DMSO in PBS. The mice in control group received the same volume of PBS. Tumor size and body weight were measured by periodic measurements with calipers every 5 days until day 45 after tumor cell implantation at which time some mice began to die. Tumor volume was calculated using the following formulae: $\mathrm{V}=$ (length $\mathrm{x}$ width $\left.{ }^{2} / 2\right)$. The survival was recorded and the mice were sacrificed at 55 days after tumor cell implantation.

Western blotting. Cells plated in 6-well plate at a density of $2 \times 10^{5}$ were treated with GRA at different concentrations for $24 \mathrm{~h}$ or cells were treated with the indicated concentrations of GRA for 2, 4, 6 and $12 \mathrm{~h}$ and total cellular proteins were extracted for western blotting. Cells were lysed in $1 \mathrm{X}$ RIPA buffer (Cell Signaling Technology, Danvers, MA, USA) containing $1 \mathrm{mM}$ phenylmethylsulfonyl fluoride (PMSF; Cell Signaling Technology) and protease inhibitor cocktail. Protein concentration was determined by BCA assay (Cwbiotech). Total proteins (20-50 $\mu \mathrm{g})$ were separated by SDS-PAGE and blotted onto to PVDF membranes. The blots were probed by antibodies against different proteins including MMP1, MMP2, MMP3, MMP9, PARP, phosphoPI3K p85/p55, phospho-AKT, AKT, phospho-STAT3, STAT3, phospho-p38, p38, phospho-Erk1/2, Erk1/2, phospho-SAPK/

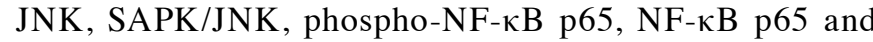
$\beta$-actin. Then the membranes were probed with horseradishperoxidase-conjugated goat anti-rabbit secondary antibody (Lianke) followed by the detection of signal with FluorChem E System (ProteinSimple, Santa Clara, CA, USA). Western blot bands were quantified by ImageJ and normalized to their respective total protein and compared with Controls (no GRA treatment).
Table I. $\mathrm{IC}_{50}(\mu \mathrm{M})$ values of GRA on different cells.

\begin{tabular}{lccc}
\hline & $24 \mathrm{~h}$ & $48 \mathrm{~h}$ & $72 \mathrm{~h}$ \\
\hline NCM460 & 866 & 144.8 & 141.3 \\
SW620 & 360.9 & 82.28 & 60.19 \\
SW480 & 153.8 & 88.23 & 34.64 \\
LoVo & 99.48 & 71.23 & 43.23 \\
\hline
\end{tabular}

Statistical analysis. All assays were performed in triplicate and experiments were repeated three times. Data are presented as mean \pm SEM. Significance of differences between the two independent groups was determined by the Student's t-test. Survival curves were drawn by the Kaplan-Meier method and analyzed using the log-rank test. Statistical calculations were performed using Graph Pad Prism. A P $<0.05$ was considered statistically significant.

\section{Results}

GRA inhibits colorectal cancer cell proliferation both in vitro and in vivo. To evaluate the general inhibitory effect of GRA on colorectal cancer cell growth, we first examined the effect of GRA on three colorectal cancer cell lines LoVo, SW620 and SW480 and the normal colon mucosal epithelial cell line NCM460. Cells were treated with GRA at varying concentrations ranging from 12.5 to $50 \mu \mathrm{M}$, as we had previously determined that the $\mathrm{IC}_{50}$ value was $>50 \mu \mathrm{M}$ in all three colorectal cancer lines and the normal colon mucosal epithelial cell line. As shown in Fig. 2A, comparing with the NCM460, the proliferation level of LoVo, SW620 and SW480 was significantly inhibited. IC $_{50}$ value of GRA for NCM460, LoVo, SW620 and SW480 cells was calculated. As detailed in Table I, the $\mathrm{IC}_{50}$ value of GRA for NCM460 cells was 866, 144.8 and $141.3 \mu \mathrm{M}$ at 24,48 and $72 \mathrm{~h}$, respectively, which is much higher than that for SW620 $(360.9,82.28$ and $60.19 \mu \mathrm{M})$, SW480 (153.8, 88.23 and 34.64 $\mu \mathrm{M})$ or LoVo $(99.48,71.23$ and $43.23 \mu \mathrm{M})$ cells. The inhibitory effect was both dose- and time-dependent. Furthermore, to evaluate whether GRA can inhibit tumorigenicity of colorectal cancer in vivo, nude mice were implanted subcutaneously with LoVo cells for 10 days followed by intraperitoneal injection of $40 \mathrm{mg} / \mathrm{kg}$ GRA or vehicle (DMSO) once every 2 days for 1 month. As seen in Fig. 2B, the tumor volume was significantly larger in the control group than that in GRA-treated group. Consistently, the body weight of the GRA-treated group decreased more slowly than the DMSO-treated group. Survival curve till 55 days after tumor cells implanted is shown in Fig. $2 \mathrm{C}$. The survival periods of LoVo xenograft mice in GRA-treated group were significantly longer than that of DMSO-treated mice $(\mathrm{P}<0.05$; Kaplan-Meier). These results show that GRA can effectively inhibit colorectal cancer development both in vitro and in vivo.

GRA promotes apoptosis in colorectal cancer cells. To investigate whether GRA inhibits cells proliferation by regulating apoptosis, Annexin V-FITC staining was performed in LoVo, SW620 and SW480 cells after treatment with GRA at 25 and 


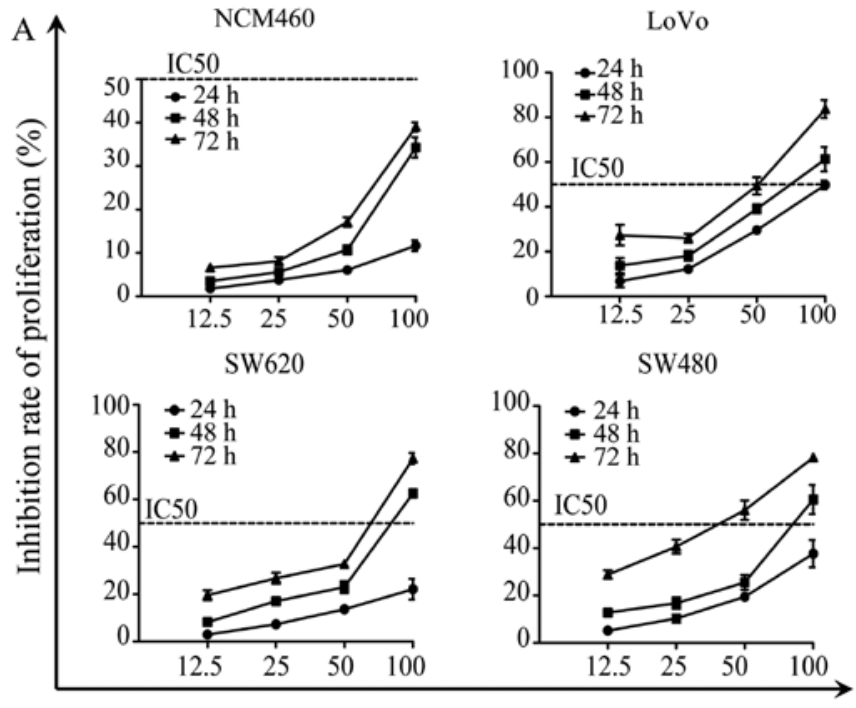

Concentration of GRA $(\mu \mathrm{M})$
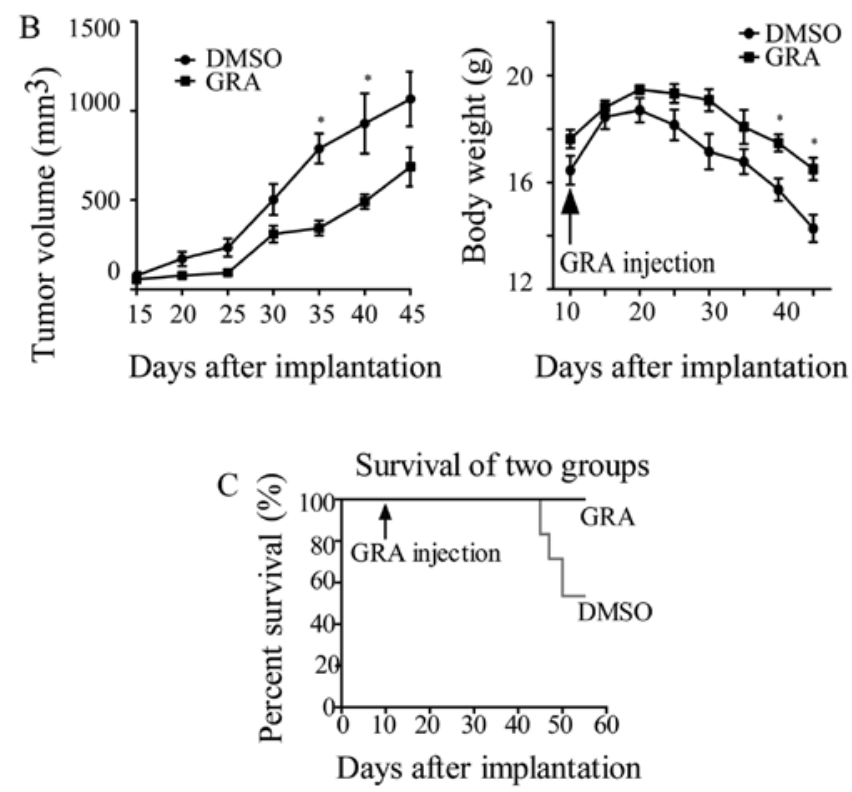

Figure 2. GRA inhibits colorectal cancer cell proliferation both in vitro and in vivo. (A) LoVo, SW620, SW480 and NCM460 cells were treated with $0,12.5,25$ and $50 \mu \mathrm{M}$ of GRA for 1,2 or 3 days followed by the CCK- 8 assay to analyze the cell growth. Absorbance at $450 \mathrm{~nm}$ was measured on an ultra-microplate reader. The growth inhibition rate was calculated as the following formula: (Ab. control - Ab. treated)/Ab. control x 100. (B) Nude mice were inoculated with LoVo cells followed by vehicle (DMSO) or $40 \mathrm{mg} / \mathrm{kg}$ GRA-treatment 10 days after inoculation. The volume of tumors and weight of the mice were measured every 5 days. Results were obtained from 3 independent experiments and expressed as means \pm SEM. ${ }^{~} \mathrm{P}<0.05$. (C) Kaplan-Meier overall survival curve of LoVo cell-implanted nude mice until day 55. The survival probability in nude mice with GRA treatment $(n=5)$ was significantly higher than that in mice treated with DMSO $(n=5)$. Log-rank test, $\mathrm{P}=0.0432$.

$50 \mu \mathrm{M}$ for $48 \mathrm{~h}$. As shown in Fig. 3A, flow cytometric analysis showed a significant increase in the apoptotic population of the cells treated with GRA compared with that of the cells without treatment. Anti-apoptotic protein survivin is expressed abundantly in human colorectal cancer and its expression indicates poor prognosis and a pro-metastatic phenotype (19). Inhibition of survivin expression is associated with the growth suppres- sion and apoptosis induction by antitumor agents (20), thus, we next investigated whether the pro-apoptotic effect of GRA was mediated by regulation of survivin expression. We detected the survivin expression in LoVo, SW620 and SW480 cells after treatment with GRA by RT-qPCR. As shown in Fig. 3B, GRA induced a dose-dependent reduction of survivin expression, of which the most significant reduction was at $50 \mu \mathrm{M}$, suggesting a potential role for survivin in GRA-induced colorectal cancer cells death. Poly-ADP-ribose polymerase (PARP), one essential substrate cleaved by both caspase- 3 and -7 , is an abundant DNA-binding enzyme. The presence of cleaved PARP-1 is one of the most potent indicators for the detection of apoptosis in many cell types $(21,22)$. Thus, we also tested it and found that the active form of PARP was slightly induced upon GRA treatment as shown in Fig. 3C. These data indicated that GRA suppresses colorectal cancer development partly through promoting the apoptosis process.

GRA inhibits the invasion and migration of colorectal cancer cells. To determine whether GRA was involved in the regulation of colorectal cancer metastasis, we performed Transwell assay on LoVo, SW620 and SW480 cells. Cells plated in the upper chamber in serum-free medium were treated with $50 \mu \mathrm{M}$ GRA for $48 \mathrm{~h}$. The invasive and migratory abilities were evaluated based on the numbers of cells passed through the membrane. As shown in Fig. 4A, in control group, the number of invaded LoVo, SW620, SW480 cells was $75.5 \pm 5.838,106.5 \pm 12.98$ and $122.3 \pm 11.74$, but in GRA treated cells, the numbers decreased to $49.50 \pm 3.663,41.75 \pm 4.385$ and $47.25 \pm 3.966$, respectively $(\mathrm{P}<0.05)$. The migration assay (Fig. 4B) showed similar results. With the number of migrated LoVo, SW620, SW480 cell being $222.8 \pm 19.44,54.25 \pm 3.425$ and $56.50 \pm 2.723$, the treatment of GRA decreased the numbers to $110 \pm 4.435,33.5 \pm 2.598$ and $35.25 \pm 3.250$, respectively $(\mathrm{P}<0.05)$. Taken together, the results indicated that GRA inhibits invasive and migratory ability of colorectal cancer cells. In addition, we also calculated the number of the LoVo cells after invasion in both sides of the chamber. As shown in Fig. 4C, GRA treatment significantly decreased LoVo cell invasion while had no significant effect on the cell numbers in the upper side of the chamber, indicating that the invasion and migration inhibitory effect of GRA was not caused by decrease of the cell number. Consistent with the invasion and migration results, GRA treatment induced a slower closing of scratch wounds in LoVo cells, indicating that GRA efficiently inhibited motility activity of LoVo cells (Fig. 4D). We next determined the changes of the expression of several matrix metalloproteinases (MMPs), which are strongly related with tumor metastasis and overexpressed in human colorectal cancers (23). MMP1, MMP2, MMP3 and MMP9 were significantly decreased after GRA treatment in LoVo, SW620 and SW480 cells (Fig. 4E). These data together clearly show that GRA could impede the invasion and migration of colorectal cancer cells.

GRA inhibits the phosphorylation of PI3K/AKT, STAT3, p38, $J N K$ and $N F-\kappa B$ in colorectal cancer cells. Previous studies have shown multiple signaling pathways such as PI3K/AKT, p-STAT3, MAPKs and NF- $\mathrm{B}$ are activated in colorectal cancers, and activation of these signaling pathways affect the proliferation, invasion and migration of tumor cells. Thus, we 


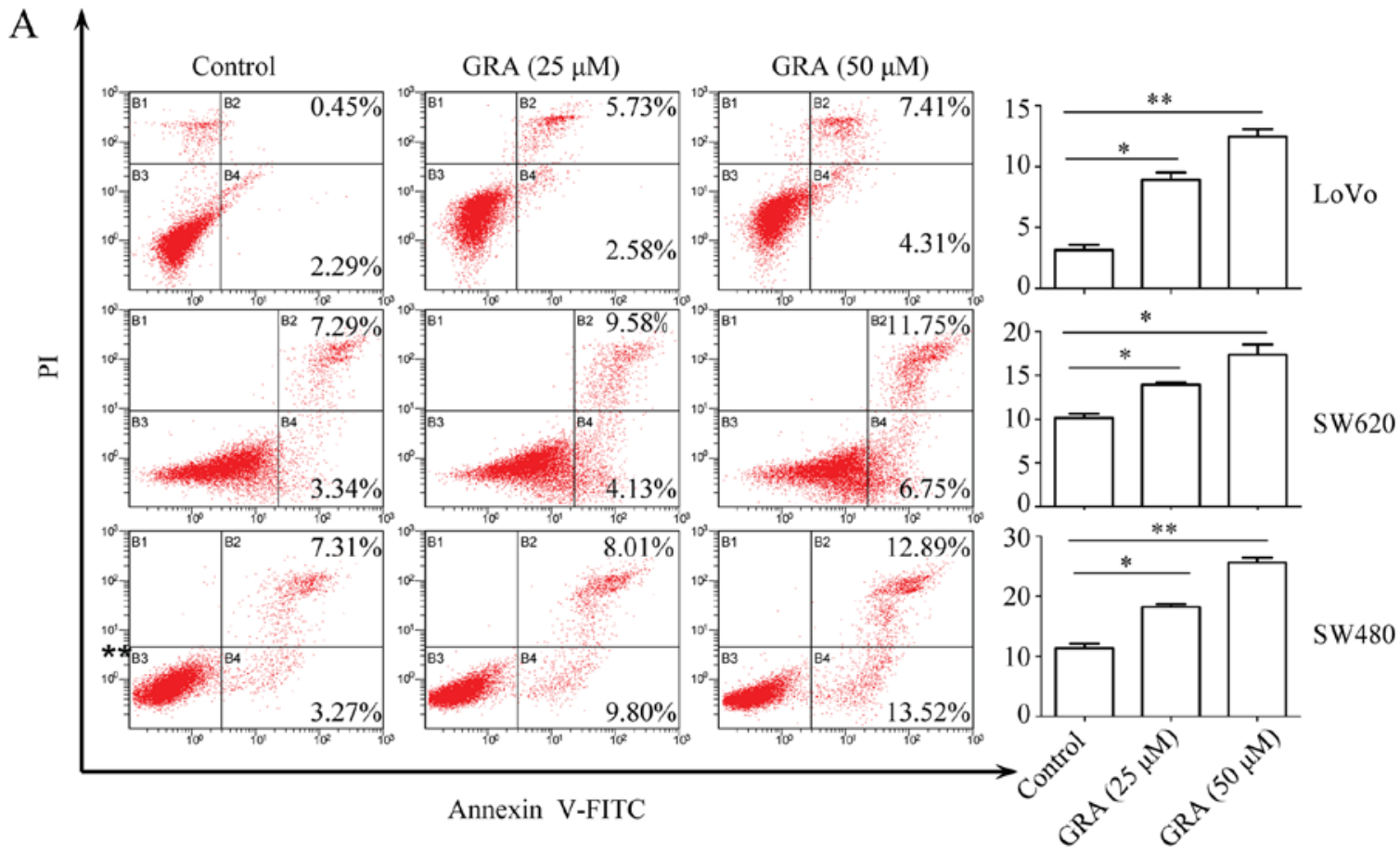

B

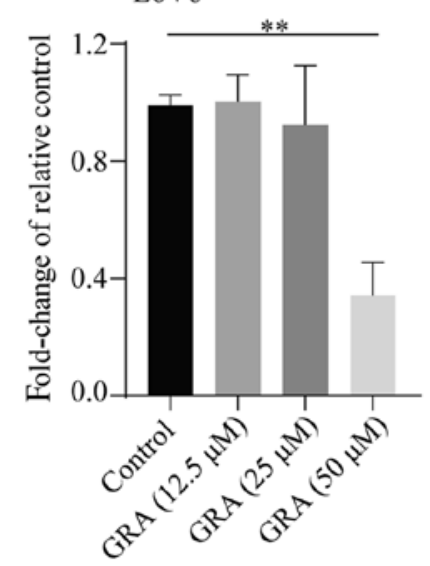

SW620

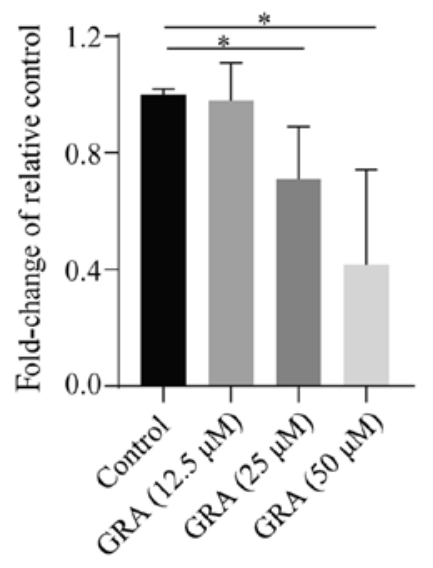

SW480

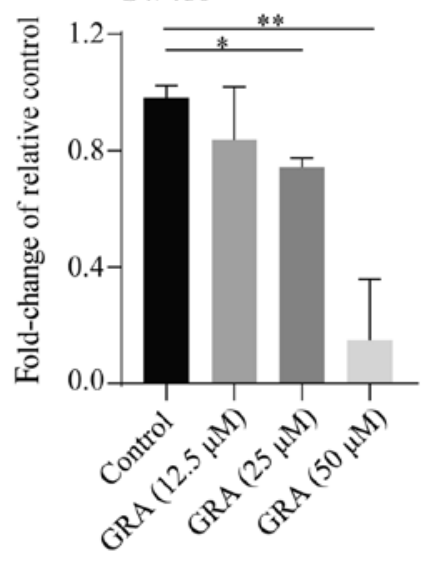

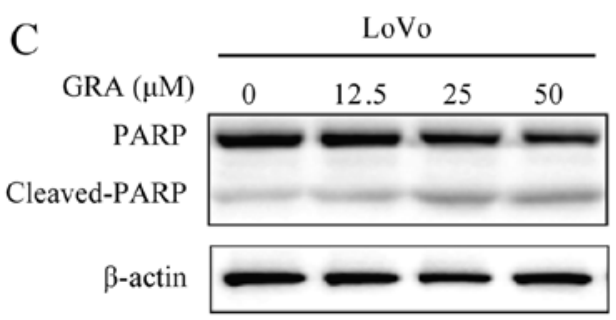
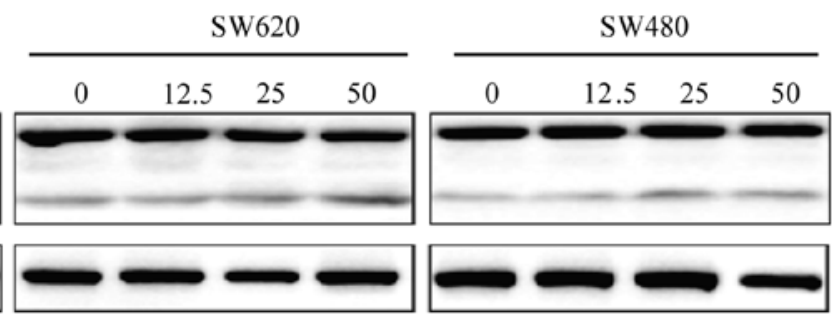

Figure 3. GRA promotes apoptosis in colorectal cancer cells. (A) Apoptosis was measured by flow cytometric analysis of Annexin V-FITC double-stained LoVo, SW620 and SW480 cells treated with GRA ( 25 or $50 \mu \mathrm{M}$ ) for $48 \mathrm{~h}$. (B) LoVo, SW620 and SW480 cells were treated with GRA at different concentrations for $24 \mathrm{~h}$ and mRNA expression of survivin was determined by RT-qPCR. $\beta$-actin was used as internal control. (C) LoVo, SW620 and SW480 cells were treated with GRA at different concentrations for $24 \mathrm{~h}$ and total cellular proteins were extracted for western blotting to detect the PARP expression. Results were obtained from 3 independent experiments and expressed as means \pm SEM. ${ }^{*} \mathrm{P}<0.05,{ }^{* *} \mathrm{P}<0.01$.

investigated whether GRA inhibited proliferation, invasion, and migration of colorectal cancer cells by altering the activation of these signaling pathways. LoVo, SW620 and SW480 cells were treated with GRA for $24 \mathrm{~h}$ before proteins were extracted for western blot analysis. As shown in Fig. 5, GRA dose-dependently reduced the protein expression of p-PI3K, 
A

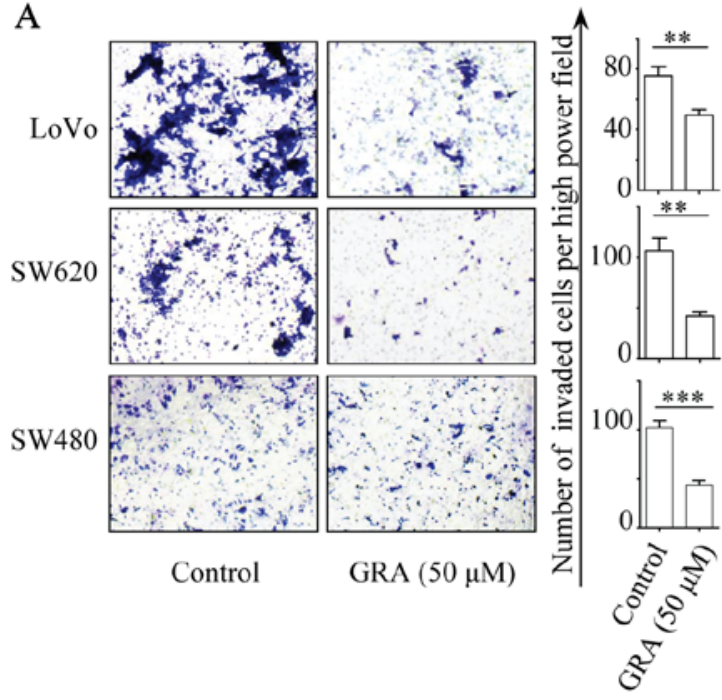

B

LoVo

SW620

SW480
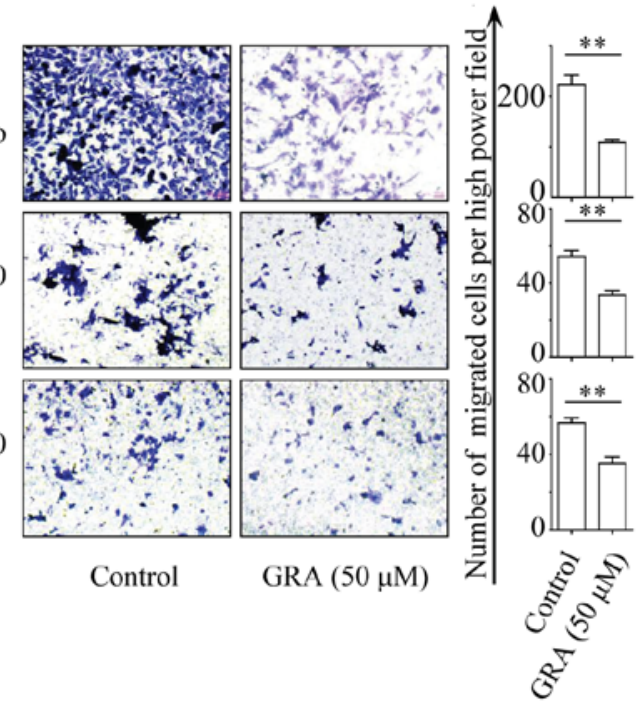

D

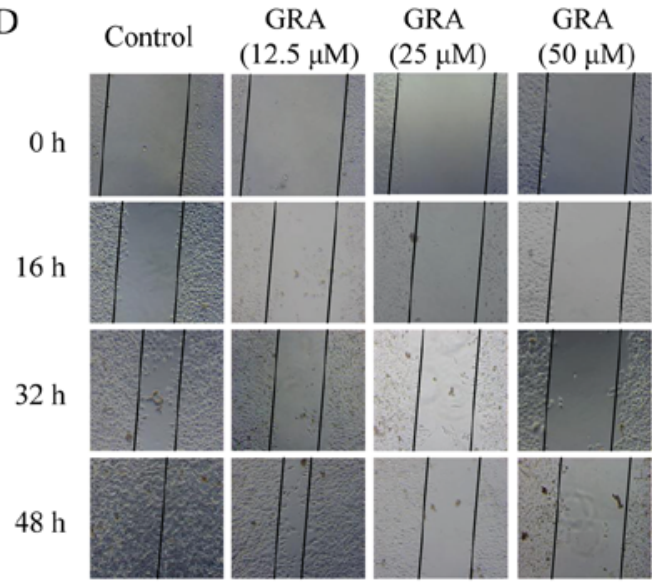

Control

= GRA (12.5 $\mu \mathrm{M})$

$=$ GRA $(25 \mu \mathrm{M})$

ॠें

20

$=$ GRA $(50 \mu \mathrm{M})$

${ }^{300}$

II I

GRA $(50 \mu \mathrm{M})$
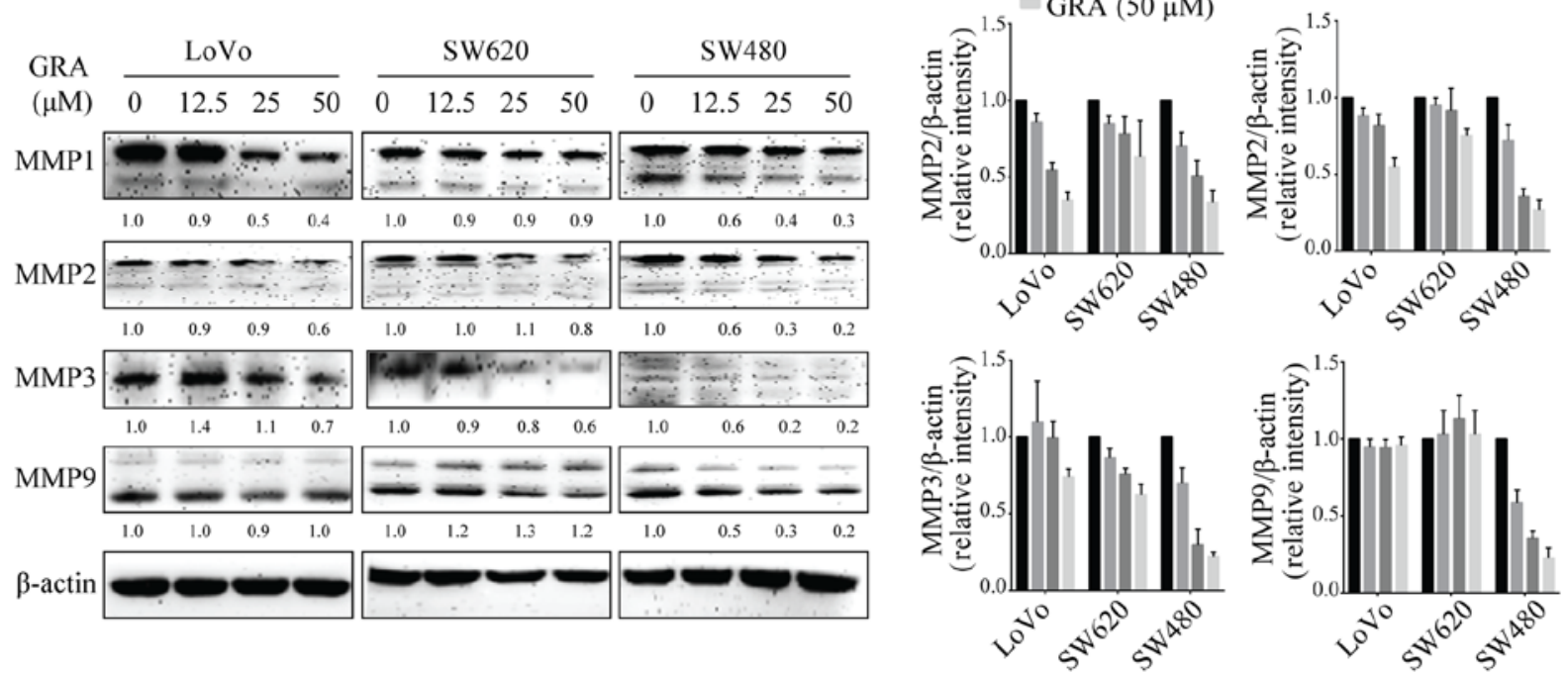

Figure 4. GRA inhibits the invasion and migration of colorectal cancer cells. Invasion and migration assays of LoVo, SW620 and SW480 cells treated with $50 \mu \mathrm{M}$ GRA or not were performed using cell culture chambers with $8-\mu \mathrm{m}$ pore filter. Forty-eight hours later, the cells invaded (A) or migrated (B) to the underside of the membrane were quantitated by cell counting in five predetermined fields. (C) Invasion of LoVo cells treated with $50 \mu \mathrm{M}$ GRA or not was performed as above. Forty-eight hours later, the cells in both sides of the chamber were determined. (D) Monolayers of LoVo cells were wounded by a plastic tip and then incubated for another $24 \mathrm{~h}$ in 5\% FBS medium with different concentrations of GRA. Images of the wound morphology were acquired by light microscopy. (E) LoVo, SW620 and SW480 cells were treated with GRA at different concentrations for $24 \mathrm{~h}$ and total cellular proteins were extracted for western blotting to detect the MMP expression. Western blot bands were quantified by ImageJ and normalized to $\beta$-actin and compared with control (no GRA treatment). Results were obtained from 3 independent experiments and expressed as means $\pm \mathrm{SEM}$. Representative images are presented. ${ }^{*} \mathrm{P}<0.05,{ }^{* *} \mathrm{P}<0.01,{ }^{* * * *} \mathrm{P}<0.001$. 
A

\begin{tabular}{|c|c|c|c|c|c|c|c|c|c|c|c|}
\hline \multirow[b]{2}{*}{ GRA $(\mu \mathrm{M})$} & \multicolumn{3}{|c|}{ LoVo } & \multicolumn{4}{|c|}{ SW620 } & \multicolumn{4}{|c|}{ SW480 } \\
\hline & 0 & 12.5 & $25 \quad 50$ & 0 & 12.5 & 525 & 50 & 0 & 12.5 & 25 & 50 \\
\hline p-AKT & 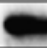 & $=$ & $-\infty$ & $=$ & $=$ & $=0$ & $=$ & & - & $=$ & $=$ \\
\hline & 1.0 & 0.5 & $\begin{array}{ll}0.5 & 0.4 \\
\end{array}$ & 1.0 & 0.8 & 0.5 & 0.5 & 1.0 & 0.6 & 0.5 & 0.3 \\
\hline
\end{tabular}

p-PI3K (p85/p55)

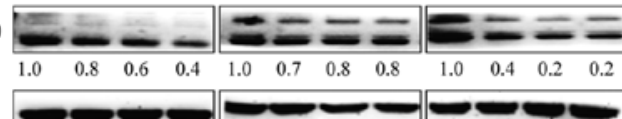

$\beta$-actin

PI3K/AKT signaling pathway

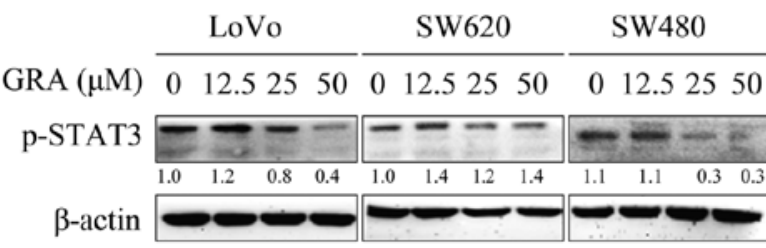

STAT3 signaling pathway

$\mathrm{C}$

\begin{tabular}{|c|c|c|c|}
\hline & LoVo & SW620 & SW480 \\
\hline GRA $(\mu \mathrm{M})$ & $012.525 \quad 50$ & $12.525 \quad 50$ & $012.525 \quad 50$ \\
\hline
\end{tabular}

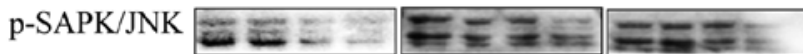

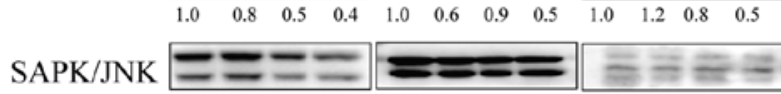
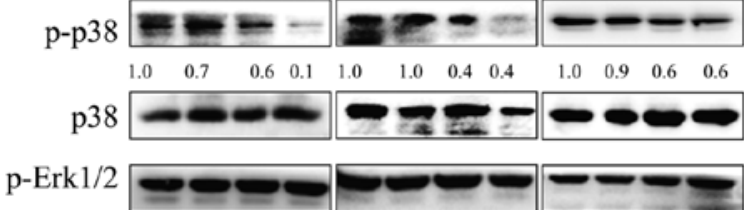

$\begin{array}{llllllllllll}1.0 & 0.9 & 0.9 & 1.0 & 1.0 & 1.1 & 0.7 & 0.8 & 1.0 & 1.0 & 0.9 & 1.0\end{array}$

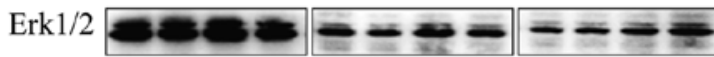

$\beta$-actin

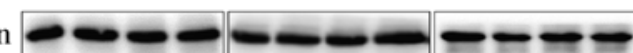

MAPK signaling pathway

D

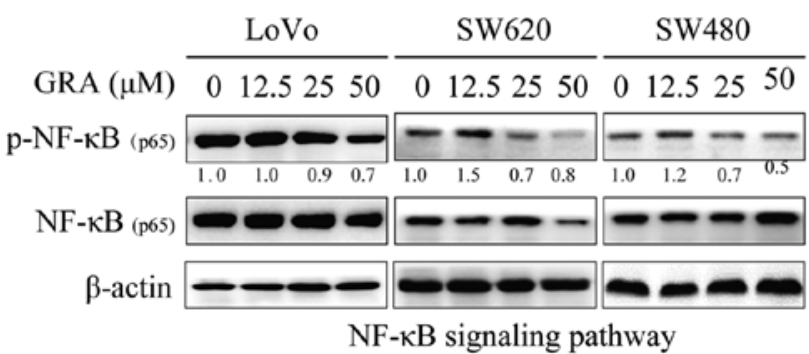

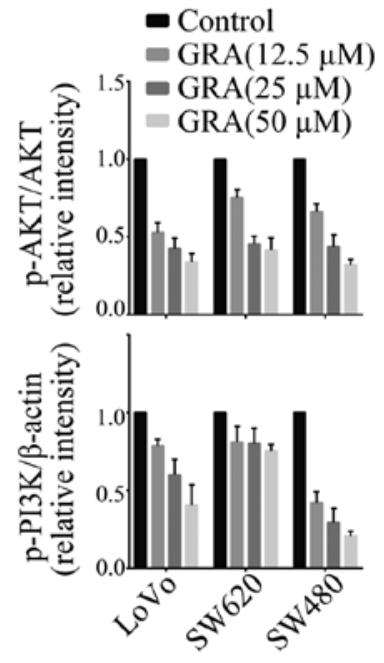
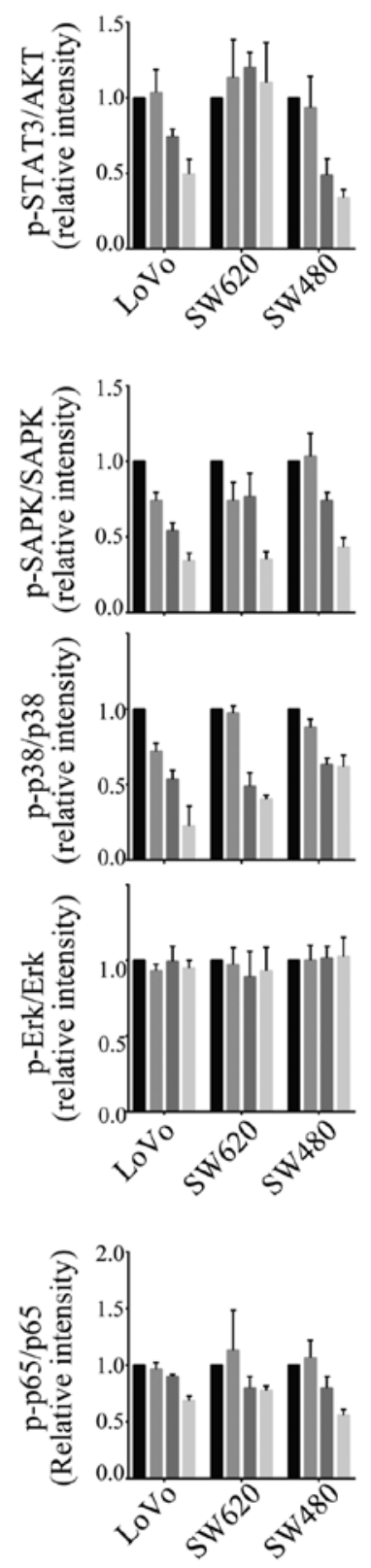

Figure 5. GRA inhibits the phosphorylation of PI3K/AKT, STAT3, p38 and JNK in colorectal cancer cells. LoVo, SW620 and SW480 cells $\left(2 \times 10^{5}\right)$ were treated with GRA at different concentrations for $24 \mathrm{~h}$ and total cellular proteins were extracted for western blotting to detect the expression of (A) p-PI3K p85/p55,

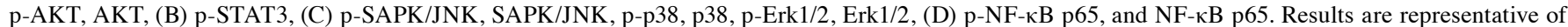
three independent experiments. Western blot bands were quantified by ImageJ and normalized to their own total proteins and compared with Control (no GRA treatment). 


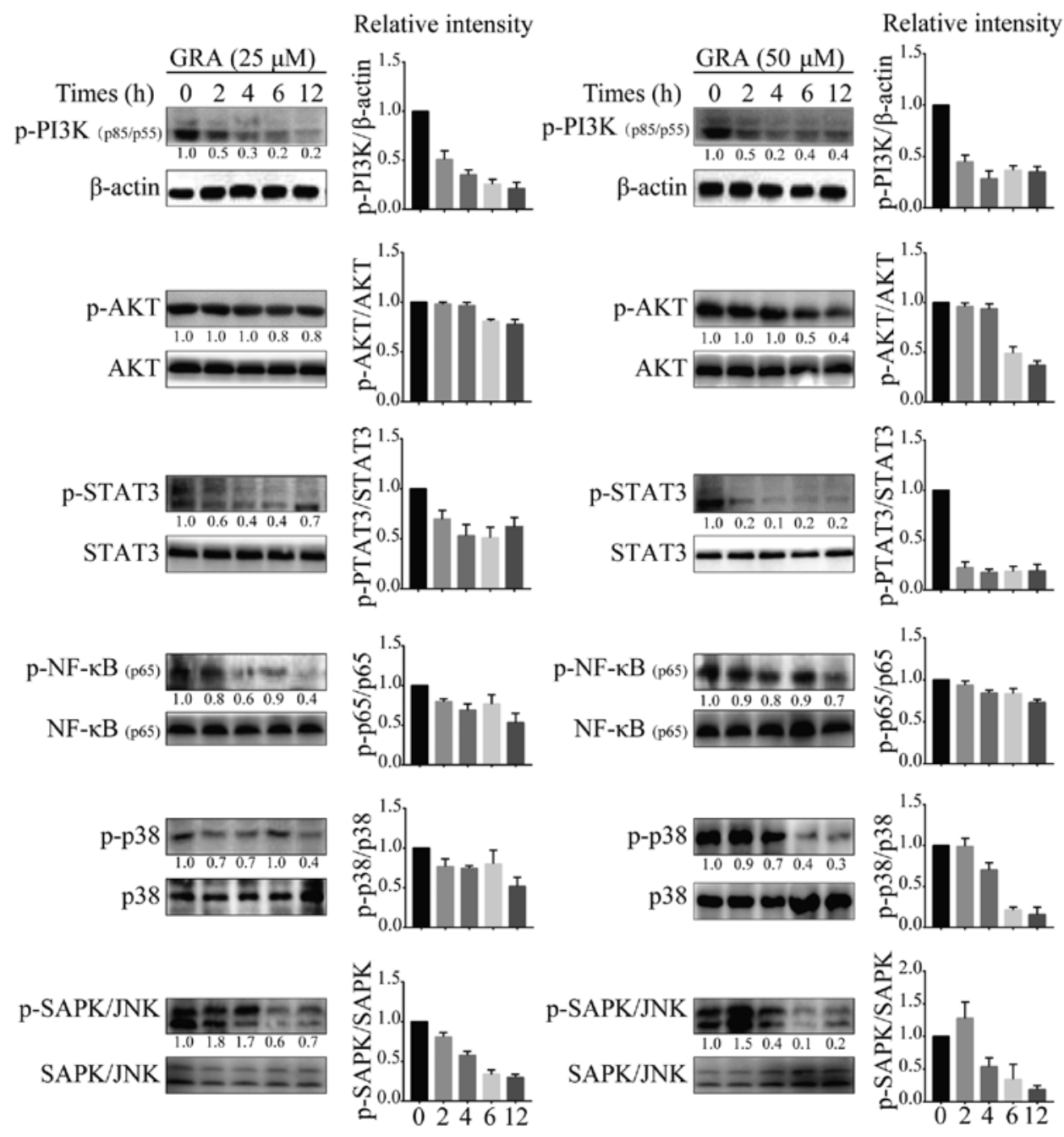

Figure 6. GRA inhibits the phosphorylation of PI3K P85/P55 and STAT3 in LoVo cells as early as $2 \mathrm{~h}$. LoVo cells $\left(2 \times 10^{5}\right)$ were treated with GRA at 25 or $50 \mu \mathrm{M}$ for $2,4,6$ or $12 \mathrm{~h}$ and total cellular proteins were extracted for western blotting to detect the expression of p-PI3K p85/p55, p-AKT, AKT, p-STAT3, STAT3, p-SAPK/JNK, SAPK/JNK, p-p38, p38, p-NF-кB p65, and NF-кB p65. Results are representative of two independent experiments. Western blot bands were quantified by ImageJ and normalized to their own total proteins and compared with control (no GRA treatment).

p-AKT, p-STAT3, p-JNK, p-p38 and p-NF-кB whereas had no significant effect on the expression of $\mathrm{p}$-Erk. Time course studies were also performed at 2, 4, 6 and $12 \mathrm{~h}$ after GRA treatment. As shown in Fig. 6, the phosphorylation levels of PI3K p85/p55 and STAT3 decreased as early as $2 \mathrm{~h}$ after GRA treatment, suggesting they are the main signaling pathways regulated by GRA. The phosphorylation levels of p-AKT, p-JNK, p-p38 and NF- $\kappa$ B p65 were also decreased, but in later time-points, suggesting these effects may be secondary to the drug treatment.

\section{Discussion}

Although great progresses have been made in developing therapeutic approaches against colorectal cancer, serious side-effects of those available treatments is still a problem beyond people's acceptance in most cases. Traditional Chinese medicine may be an untapped treasure for developing better agents for cancer remedy since more and more Chinese herbs are found to efficiently suppress various cancers with lower side-effects. In the present study, we report that GRA, a component of Radix Glycyrrbiza with the antitumor activity against human breast cancer cell lines (7) and lung cancer cell line (24) inhibits the proliferation, migration and invasion of colorectal cancer cell lines LoVo, SW620 and SW480. Furthermore, our results indicated that GRA-induced apoptosis as well as downregulation of MMP1, 2, 3, 9 expression, respectively, is associated with the downregulation of PI3K and STAT3 signaling pathways.

Activation of PI3K/AKT signaling pathway in cancer results in increased cell survival, outgrowth and enhanced cell migration (25). Inhibition of its activation will downregulate expression of survivin, MMP1, 2, 3, 9, 12, 13 and MT1-MMP, thus, suppresses the proliferation, migration and invasion of cancer cells (26-31). Our results showed that GRA treatment significantly suppresses the phosphorylation of PI3K and AKT in LoVo, SW620 and SW480 cells, and the phosphorylation of PI3K occurs as early as $2 \mathrm{~h}$ after GRA treatment. While Kao et al (32) showed that glycyrrhizic acid (GA) not GRA activates PI3K/AKT in RAW264.7 cells. There is no previous report showing that GRA could suppress the PI3K/AKT signaling activation.

Constitutive activation of STAT3 is frequently detected in primary human colorectal cancers and established colorectal 
cancer cell lines (33), and is closely related to tumor proliferation, differentiation, invasion and metastasis $(34,35)$. Elevated levels of STAT3 phosphorylation were correlated with the induced survivin gene expression and inhibition of STAT3 activation could reduce survivin expression and promote apoptosis of cancer cells $(36,37)$. Moreover, activation of STAT3 induced MMP1, 3, 7 and 9 expressions, promoting invasiveness of cancer cells (38). Our results showed that GRA treatment inhibited survivin expression and STAT3 phosphorylation in colorectal cancer cells, which is consistent with the previous study that glycyrrhizic acid inhibits the cell growth and migration via blocking AKT/STAT3 singling pathway in leukemia cells (39).

Three major mitogen activated protein kinase (MAPK) families: Erk, p38 and JNK are deregulated in colorectal cancers, and are significantly associated with cell proliferation, migration and invasion (40). Survivin is a downstream effector of p38 (41) and Erk (42). MMP production is also tightly regulated by the MAPKs (43). Phosphorylation of p38, Erk and JNK could induce MMP1, 2, 3 and 9 expression (44-53). Our results confirmed that p-Erk, p-p38 and p-JNK are activated in LoVo, SW620 and SW480 cells, while GRA treatment selectively inhibited the phosphorylation of p-p38 and p-JNK. One study showed that in breast cancer cells, GRA decreases the phosphorylation level of p38 MAPK whereas exhibits little effect on the phosphorylation levels of Erk and JNK, and subsequently decreases the MMP2 and MMP9 expression (7), suggesting that GRA presents antitumor mechanisms dependent on the origin of the cell lines.

$\mathrm{NF}-\kappa \mathrm{B}$ activation contributes to the induction of cellular transformation, progression and metastasis of colorectal cancers (54). NF- $\kappa$ B cooperates with other molecules such as STAT3, PI3K to regulate a number of target genes including survivin and MMPs (55-57). Our results showed that GRA inhibits NF- $\mathrm{BB}$ activation in LoVo, SW620 and SW480 cells, which was consistent with previous results (4). While the decreased phosphorylation of NF- $\mathrm{B}$ occurs later than STAT3 and PI3K, implying NF- $\mathrm{B}$ downregulation is secondary effect of the drug treatment.

In summary, to the best of our knowledge, we are the first to demonstrate that GRA can effectively inhibit colorectal cancer cell proliferation, invasion and migration probably through suppressing PI3K and STAT3 signaling pathways. The present study indicated that GRA could be a potential effective and safe therapeutic agent for patients with colorectal cancer.

\section{Acknowledgements}

We thank Ruoque Mao from Ulink College of Shanghai for her contributions in the CCK-8 assay as parts of her high school students training programme. The present study was supported by a grant from the Key Project of Natural Science Foundation of Zhejiang Province (LZ16H160003 to W.-B.C.).

\section{References}

1. Cao D, Jiang J, You L, Jia Z, Tsukamoto T, Cai H, Wang S, Hou Z, Suo YE and Cao X: The protective effects of $18 \beta$-glycyrrhetinic acid on Helicobacter pylori-infected gastric mucosa in Mongolian gerbils. BioMed Res Int 2016: 4943793, 2016.
2. Darvishi B, Manoochehri S, Kamalinia G, Samadi N, Amini M, Mostafavi SH, Maghazei S, Atyabi F and Dinarvand R: preparation and antibacterial activity evaluation of $18-\beta$-glycyrrhetinic acid loaded PLGA nanoparticles. Iran J Pharm Res 14: 373-383, 2015.

3. Hasan SK, Khan R, Ali N, Khan AQ, Rehman MU, Tahir M, Lateef A, Nafees S, Mehdi SJ, Rashid S, et al: 18- $\beta$ Glycyrrhetinic acid alleviates 2-acetylaminofluorene-induced hepatotoxicity in Wistar rats: Role in hyperproliferation, inflammation and oxidative stress. Hum Exp Toxicol 34: 628-641, 2015.

4. Wang CY, Kao TC, Lo WH and Yen GC: Glycyrrhizic acid and $18 \beta$-glycyrrhetinic acid modulate lipopolysaccharide-induced inflammatory response by suppression of NF- $\kappa \mathrm{B}$ through PI3K p110 $\delta$ and p110 $\gamma$ inhibitions. J Agric Food Chem 59: 7726-7733, 2011.

5. Hardy ME, Hendricks JM, Paulson JM and Faunce NR: $18 \beta$-glycyrrhetinic acid inhibits rotavirus replication in culture. Virol J 9: 96, 2012.

6. Yano S, Harada M, Watanabe K, Nakamaru K, Hatakeyama Y, Shibata S, Takahashi K, Mori T, Hirabayashi K, Takeda M, et al: Antiulcer activities of glycyrrhetinic acid derivatives in experimental gastric lesion models. Chem Pharm Bull (Tokyo) 37: 2500-2504, 1989.

7. Wang XF, Zhou QM, Lu YY, Zhang H, Huang S and Su SB: Glycyrrhetinic acid potently suppresses breast cancer invasion and metastasis by impairing the p38 MAPK-AP1 signaling axis. Expert Opin Ther Targets 19: 577-587, 2015.

8. Sharma G, Kar S, Palit S and Das PK: $18 \beta$-glycyrrhetinic acid induces apoptosis through modulation of $\mathrm{Akt} / \mathrm{FOXO} \mathrm{a} / \mathrm{Bim}$ pathway in human breast cancer MCF-7 cells. J Cell Physiol 227: 1923-1931, 2012.

9. Haghshenas V, Fakhari S, Mirzaie S, Rahmani M, Farhadifar F, Pirzadeh S and Jalili A: Glycyrrhetinic acid inhibits cell growth and induces apoptosis in ovarian cancer a2780 cells. Adv Pharm Bull 4 (Suppl 1): 437-441, 2014.

10. Pirzadeh S, Fakhari S, Jalili A, Mirzai S, Ghaderi B and Haghshenas V: Glycyrrhetinic acid induces apoptosis in leukemic HL60 cells through upregulating of CD95/ CD178. Int J Mol Cell Med 3: 272-278, 2014

11. Hibasami H, Iwase H, Yoshioka K and Takahashi H: Glycyrrhetic acid (a metabolic substance and aglycon of glycyrrhizin) induces apoptosis in human hepatoma, promyelotic leukemia and stomach cancer cells. Int J Mol Med 17: 215-219, 2006.

12. Yamaguchi H, Noshita T, Yu T, Kidachi Y, Kamiie K, Umetsu H and Ryoyama K: Novel effects of glycyrrhetinic acid on the central nervous system tumorigenic progenitor cells: Induction of actin disruption and tumor cell-selective toxicity. Eur J Med Chem 45: 2943-2948, 2010.

13. Favoriti P, Carbone G, Greco M, Pirozzi F, Pirozzi RE and Corcione F: Worldwide burden of colorectal cancer: A review. Updates Surg 68: 7-11, 2016.

14. Arvelo F, Sojo F and Cotte C: Biology of colorectal cancer. E Cancer Med Sci 9: 520, 2015.

15. Lombardi L, Morelli F, Cinieri S, Santini D, Silvestris N, Fazio N, Orlando L, Tonini G, Colucci G and Maiello E: Adjuvant colon cancer chemotherapy: Where we are and where we'll go. Cancer Treat Rev 36 (Suppl 3): S34-S41, 2010.

16. Khan K, Wale A, Brown G and Chau I: Colorectal cancer with liver metastases: Neoadjuvant chemotherapy, surgical resection first or palliation alone? World J Gastroenterol 20: 12391-12406, 2014.

17. Roohbakhsh A, Iranshahy M and Iranshahi M: Glycyrrhetinic acid and its derivatives: Anti-cancer and cancer chemopreventive properties, mechanisms of action and structure - cytotoxic activity relationship. Curr Med Chem 23: 498-517, 2016.

18. Liu J, Wang S, Zhang Y, Fan HT and Lin HS: Traditional Chinese medicine and cancer: History, present situation, and development. Thorac Cancer 6: 561-569, 2015.

19. Krieg A, Werner TA, Verde PE, Stoecklein NH and Knoefel WT: Prognostic and clinicopathological significance of survivin in colorectal cancer: A meta-analysis. PLoS One 8: e65338, 2013.

20. Groner B and Weiss A: Targeting survivin in cancer: novel drug development approaches. BioDrugs 28: 27-39, 2014.

21. Decker P and Muller S: Modulating poly (ADP-ribose) polymerase activity: Potential for the prevention and therapy of pathogenic situations involving DNA damage and oxidative stress. Curr Pharm Biotechnol 3: 275-283, 2002. 
22. Koh DW, Dawson TM and Dawson VL: Mediation of cell death by poly(ADP-ribose) polymerase-1. Pharmacol Res 52: 5-14, 2005.

23. Zucker S and Vacirca J: Role of matrix metalloproteinases (MMPs) in colorectal cancer. Cancer Metastasis Rev 23: 101-117, 2004.

24. Yadav DK, Kalani K, Khan F and Srivastava SK: QSAR and docking based semi-synthesis and in vitro evaluation of 18 $\beta$-glycyrrhetinic acid derivatives against human lung cancer cell line A-549. Med Chem 9: 1073-1084, 2013.

25. Manning BD and Cantley LC: AKT/PKB signaling: Navigating downstream. Cell 129: 1261-1274, 2007.

26. Wang J, Yang L, Yang J, Kuropatwinski K, Wang W, Liu XQ, Hauser $\mathbf{J}$ and Brattain MG: Transforming growth factor beta induces apoptosis through repressing the phosphoinositide 3-kinase/AKT/survivin pathway in colon cancer cells. Cancer Res 68: 3152-3160, 2008.

27. Zhang B, Leng C, Wu C, Zhang Z, Dou L, Luo X, Zhang B and Chen X: Smad4 sensitizes colorectal cancer to 5-fluorouracil through cell cycle arrest by inhibiting the PI3K/Akt/CDC2/ survivin cascade. Oncol Rep 35: 1807-1815, 2016.

28. Zhang X, Shi H, Tang H, Fang Z, Wang J and Cui S: miR-218 inhibits the invasion and migration of colon cancer cells by targeting the PI3K/Akt/mTOR signaling pathway. Int J Mol Med 35: 1301-1308, 2015.

29. Chen JS, Wang Q, Fu XH, Huang XH, Chen XL, Cao LQ, Chen LZ, Tan HX, Li W, Bi J, et al: Involvement of PI3K/PTEN/ $\mathrm{AKT} / \mathrm{mTOR}$ pathway in invasion and metastasis in hepatocellular carcinoma: Association with MMP-9. Hepatol Res 39: 177-186, 2009

30. Liu M, Hu Y, Zhang MF, Luo KJ, Xie XY, Wen J, Fu JH and Yang H: MMP1 promotes tumor growth and metastasis in esophageal squamous cell carcinoma. Cancer Lett 377: 97-104, 2016.

31. Ito $\mathrm{S}$, Kimura $\mathrm{K}$, Haneda $\mathrm{M}$, Ishida $\mathrm{Y}$, Sawada $\mathrm{M}$ and Isobe $\mathrm{K}$ : Induction of matrix metalloproteinases (MMP3, MMP12 and MMP13) expression in the microglia by amyloid-beta stimulation via the PI3K/Akt pathway. Exp Gerontol 42: 532-537, 2007.

32. Kao TC, Shyu MH and Yen GC: Glycyrrhizic acid and 18betaglycyrrhetinic acid inhibit inflammation via PI3K/Akt/GSK3beta signaling and glucocorticoid receptor activation. J Agric Food Chem 58: 8623-8629, 2010.

33. Ma XT, Wang S, Ye YJ, Du RY, Cui ZR and Somsouk M: Constitutive activation of Stat 3 signaling pathway in human colorectal carcinoma. World J Gastroenterol 10: 1569-1573, 2004.

34. Xiong H, Zhang ZG, Tian XQ, Sun DF, Liang QC, Zhang YJ, Lu R, Chen YX and Fang JY: Inhibition of JAK1, 2/STAT3 signaling induces apoptosis, cell cycle arrest, and reduces tumor cell invasion in colorectal cancer cells. Neoplasia 10: 287-297, 2008.

35. Wang SW and Sun YM: The IL-6/JAK/STAT3 pathway: Potential therapeutic strategies in treating colorectal cancer (Review). Int J Oncol 44: 1032-1040, 2014.

36. Lassmann S, Schuster I, Walch A, Göbel H, Jütting U, Makowiec F, Hopt U and Werner M: STAT3 mRNA and protein expression in colorectal cancer: Effects on STAT3-inducible targets linked to cell survival and proliferation. J Clin Pathol 60 : 173-179, 2007

37. Lin L, Liu A, Peng Z, Lin HJ, Li PK, Li C and Lin J: STAT3 is necessary for proliferation and survival in colon cancer-initiating cells. Cancer Res 71: 7226-7237, 2011.

38. Tsareva SA, Moriggl R, Corvinus FM, Wiederanders B, Schütz A, Kovacic B and Friedrich K: Signal transducer and activator of transcription 3 activation promotes invasive growth of colon carcinomas through matrix metalloproteinase induction. Neoplasia 9: 279-291, 2007.

39. He SQ, Gao M, Fu YF and Zhang YN: Glycyrrhizic acid inhibits leukemia cell growth and migration via blocking AKT/mTOR/ STAT3 signaling. Int J Clin Exp Pathol 8: 5175-5181, 2015.
40. Urosevic J, Nebreda AR and Gomis RR: MAPK signaling control of colon cancer metastasis. Cell Cycle 13: 2641-2642, 2014.

41. Cui WJ, Liu Y, Zhou XL, Wang FZ, Zhang XD and Ye LH: Myosin light chain kinase is responsible for high proliferative ability of breast cancer cells via anti-apoptosis involving p38 pathway. Acta Pharmacol Sin 31: 725-732, 2010.

42. Siddiqa A, Long LM, Li L, Marciniak RA and Kazhdan I: Expression of HER-2 in MCF-7 breast cancer cells modulates anti-apoptotic proteins survivin and Bcl-2 via the extracellular signal-related kinase (ERK) and phosphoinositide-3 kinase (PI3K) signalling pathways. BMC Cancer 8: 129, 2008.

43. Reuben PM and Cheung HS: Regulation of matrix metalloproteinase (MMP) gene expression by protein kinases. Front Biosci 11: 1199-1215, 2006

44. Uhlirova $M$ and Bohmann D: JNK- and Fos-regulated Mmp1 expression cooperates with Ras to induce invasive tumors in Drosophila. EMBO J 25: 5294-5304, 2006.

45. Davidson B, Givant-Horwitz V, Lazarovici P, Risberg B, Nesland JM, Trope CG, Schaefer E and Reich R: Matrix metalloproteinases (MMP), EMMPRIN (extracellular matrix metalloproteinase inducer) and mitogen-activated protein kinases (MAPK): Co-expression in metastatic serous ovarian carcinoma. Clin Exp Metastasis 20: 621-631, 2003.

46. Chen F, Deng J, Liu X, Li W and Zheng J: HCRP-1 regulates cell migration and invasion via EGFR-ERK mediated up-regulation of MMP-2 with prognostic significance in human renal cell carcinoma. Sci Rep 5: 13470, 2015.

47. Song KH, Kim SH, Noh KH, Bae HC, Kim JH, Lee HJ, Song J, Kang TH, Kim DW, Oh SJ, et al: Apoptosis inhibitor 5 increases metastasis via Erk-mediated MMP expression. BMB Rep 48: 330-335, 2015.

48. Liu Z, Cai H, Zheng X, Zhang B and Xia C: The involvement of mutual inhibition of ERK and mTOR in PLC $\gamma 1$-mediated MMP-13 expression in human osteoarthritis chondrocytes. Int $\mathrm{J}$ Mol Sci 16: 17857-17869, 2015.

49. Cho JS, Kang JH, Um JY, Han IH, Park IH and Lee HM: Lipopolysaccharide induces pro-inflammatory cytokines and MMP production via TLR4 in nasal polyp-derived fibroblast and organ culture. PLoS One 9: e90683, 2014.

50. Hammaker DR, Boyle DL, Inoue T and Firestein GS: Regulation of the JNK pathway by TGF-beta activated kinase 1 in rheumatoid arthritis synoviocytes. Arthritis Res Ther 9: R57, 2007.

51. Fromigué O, Hamidouche $\mathrm{Z}$ and Marie PJ: Blockade of the RhoA-JNK-c-Jun-MMP2 cascade by atorvastatin reduces osteosarcoma cell invasion. J Biol Chem 283: 30549-30556, 2008.

52. Johnson GL and Lapadat R: Mitogen-activated protein kinase pathways mediated by ERK, JNK, and p38 protein kinases. Science 298: 1911-1912, 2002.

53. Sinfield JK, Das A, O'Regan DJ, Ball SG, Porter KE and Turner NA: p38 MAPK alpha mediates cytokine-induced IL-6 and MMP-3 expression in human cardiac fibroblasts. Biochem Biophys Res Commun 430: 419-424, 2013.

54. Wang S, Liu Z, Wang L and Zhang X: NF-kappaB signaling pathway, inflammation and colorectal cancer. Cell Mol Immunol 6: 327-334, 2009

55. Wang K, Brems JJ, Gamelli RL and Holterman AX: Survivin signaling is regulated through nuclear factor-kappa B pathway during glycochenodeoxycholate-induced hepatocyte apoptosis. Biochim Biophys Acta 1803: 1368-1375, 2010.

56. Hoesel B and Schmid JA: The complexity of NF- $\kappa B$ signaling in inflammation and cancer. Mol Cancer 12: 86, 2013

57. Li W, Wang H, Kuang CY, Zhu JK, Yu Y, Qin ZX, Liu J and Huang L: An essential role for the Id1/PI3K/Akt/NFkB/survivin signalling pathway in promoting the proliferation of endothelial progenitor cells in vitro. Mol Cell Biochem 363: 135-145, 2012. 